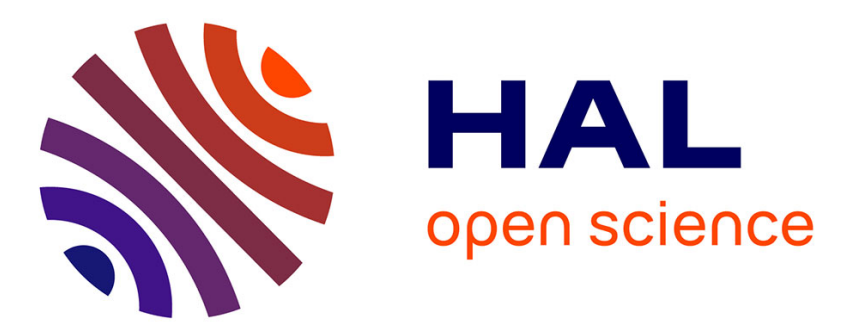

\title{
Utilisation d'un seul dioptre et d'un analyseur ellipsomètre interférentiel pour l'amélioration des mesures d'indices par réflecto-ellipsométrie en incidence brewstérienne ou principale
}

R. Sardos

\section{To cite this version:}

R. Sardos. Utilisation d'un seul dioptre et d'un analyseur ellipsomètre interférentiel pour l'amélioration des mesures d'indices par réflecto-ellipsométrie en incidence brewstérienne ou principale. Revue de Physique Appliquée, 1974, 9 (5), pp.947-951. 10.1051/rphysap:0197400905094700 . jpa-00243860

\author{
HAL Id: jpa-00243860 \\ https://hal.science/jpa-00243860
}

Submitted on 1 Jan 1974

HAL is a multi-disciplinary open access archive for the deposit and dissemination of scientific research documents, whether they are published or not. The documents may come from teaching and research institutions in France or abroad, or from public or private research centers.
L'archive ouverte pluridisciplinaire HAL, est destinée au dépôt et à la diffusion de documents scientifiques de niveau recherche, publiés ou non, émanant des établissements d'enseignement et de recherche français ou étrangers, des laboratoires publics ou privés. 


\title{
UTILISATION D'UN SEUL DIOPTRE \\ ET D'UN ANALYSEUR ELLIPSOMĖTRE INTERFÉRENTIEL POUR L'AMÉLIORATION DES MESURES D'INDICES PAR RÉFLECTO-ELLIPSOMÉTRIE EN INCIDENCE BREWSTÉRIENNE OU PRINCIPALE
}

\author{
R. SARDOS \\ Laboratoire de Physique Expérimentale, Equipe d'optique des micro-ondes, \\ Université de Bordeaux I, 351, Cours de la Libération, 33405 Talence, France
}

(Reçu le 25 mars 1974, révisé le 13 mai 1974)

\begin{abstract}
Résumé. - 1) L'auteur montre que, dans tout le spectre électromagnétique, il est aisé de s'affranchir des réflexions parasites en utilisant une lame de forme convenable.

2) Il montre ensuite que l'on peut également améliorer les mesures en micro-ondes à l'aide d'un analyseur ellipsomètre différent de ceux classiquement utilisés.

Abstract. - The author shows that throughout the whole electromagnetic spectrum it is easy to suppress parasitic reflections by means of a judiciously shaped sample.

He then shows it is also possible to improve the measurements in the microwave region with an unusual type of ellipsometer.
\end{abstract}

Les mesures d'indices, ou de constantes diélectriques, à l'aide de l'incidence brewstérienne ou de l'incidence principale présentent un intérêt certain pour les corps portés à haute température par exemple [1], et dans certains domaines de fréquences, car les mesures se font à l'air libre. Elles se heurtent toutefois à quelques difficultés expérimentales. La première provient des réflexions multiples à l'intérieur de la lame, la seconde est inhérente à l'analyseur utilisé.

1. Elimination des erreurs provenant des réflexions multiples à travers la lame. - On peut montrer partant des formules d'Airy [2] qu'il y aurait lieu d'introduire pour le calcul des réflexions multiples deux épaisseurs équivalentes (compte tenu des sauts de phase à la réflexion) l'une pour les vibrations situées dans le plan d'incidence, l'autre pour les vibrations perpendiculaires à ce plan. Cette difficulté est surmontée dans le cas de corps très absorbants [1] en prenant une lame d'épaisseur assez grande de façon à pouvoir négliger les réflexions multiples. On montre que si

$$
l \geqslant \frac{1}{\alpha} \quad \text { avec } \quad \alpha=\frac{2 \pi}{\lambda} \sqrt{|\varepsilon|} \sin \delta / 2
$$

( $\delta$, angle de perte) les erreurs dues aux réflexions multiples sont inférieures à $1 / 100^{\mathrm{e}}$; encore faut-il connaître les valeurs approchées de $\varepsilon$ et de $\delta$. Pour les corps transparents ou très peu absorbants il $\mathrm{y}$ a lieu d'effec- tuer un calcul de correction peu passionnant (souvent simplifié en prenant une seule épaisseur équivalente). Ce calcul de correction est d'ailleurs illusoire si la mesure de l'épaisseur de la lame (difficile dans le cas des liquides) n'a pas été faite avec une précision suffisante.

Il semble plus simple et plus rationnel d'éliminer l'influence des réflexions multiples et par suite l'épaisseur de la lame en n'utilisant qu'un seul dioptre. L'adaptation de la seconde face déjà employée dans un cas voisin [3,4] est à exclure puisqu'on ignore l'indice de l'échantillon. Dans le cas d'un corps transparent (cas où les réflexions multiples sont les plus gênantes) lors de la mesure de l'angle de Brewster $i_{\mathrm{B}}$, le rayon réfracté $R_{2}$ et le rayon réfléchi $R_{3}$ sur le dioptre sont orthogonaux (Fig. 1a). Si la lame est prismatique, d'angle $\pi / 2-i_{\mathrm{B}}$; le rayon réfracté arrive en $P$, sur le second dioptre, sous incidence normale.

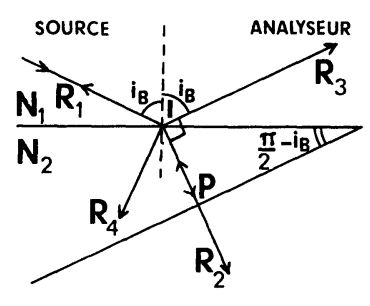

a)

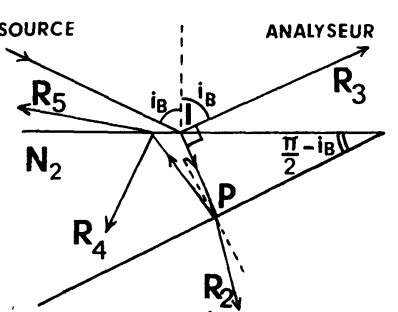

b)
FIG. 1. 
L'onde elliptique qui arrive en $P$ est partiellement réfléchie ; la partie qui revient en I change de polarisation en se réfléchissant en direction $R_{4}$ et en se réfractant en direction de la source $R_{1}$, etc... Pour que le second dioptre ne modifie en rien la réflexion sur le premier il suffit d'éliminer les rayons qui vont en direction $R_{2}$, ceux qui vont en direction $R_{4}$ et de s'assurer que la source ne réfléchit pas. On éliminera les rayons gênants en utilisant des absorbants et la géométrie de l'appareil (cas du rayon $R_{4}$ qui arrive finalement sur la base du prisme). Si la source risque de réfléchir partiellement on donnera à la lame un angle légèrement différent de $\pi / 2-i_{\mathrm{B}}$ et on voit sur la figure $1 b$ que le problème sera résolu en absorbant également le rayon $R_{5}$.

Il convient de remarquer d'une part, que l'on peut faire ainsi des mesures absolues, sans aucune correction si le premier milieu est le vide, et d'autre part, que les considérations précédentes sont valables non seulement dans tout le domaine des ondes électro-magnétiques mais également dans d'autres cas de propagation de mouvements vibratoires.

Bien entendu dans certains domaines (optique visible par exemple) il conviendra de discuter les indices obtenus en tenant compte du fait que les couches au voisinage de la surface ont un indice qui peut être différent de celui de la matière massive ; mais, dans le cas des hyperfréquences les couches étant très minces par rapport à la longueur d'onde ce phénomène sera négligeable.

En optique hertzienne la méthode de l'incidence principale (ou brewstérienne) est surtout intéressante :

a) dans le domaine centimétrique pour les cas où les méthodes en guides d'ondes sont difficilement utilisables [1],

b) dans la gamme millimétrique car les méthodes en ondes guidées sont alors entachées d'erreurs nombreuses (remplissage du guide, etc...).

Pour les liquides on réalisera une cuve déformable (accordéon) à niveau constant, la face plane et horizontale constituant le dioptre de mesure, le fond de la cuve restant approximativement parallèle (pour éviter les réflexions dues à la source) à la direction de l'analyseur, qui suit la direction du rayon réfléchi grâce à un pseudo-parallélogramme.

Dans le cas des solides on fait une première mesure rapide qui permet d'obtenir un indice approché, puis on taille ensuite une lame prismatique. L'angle de cette lame est proche de la valeur optimum et satisfait alors automatiquement à la condition : incidence sur le second dioptre quasi-normale.

2. Amélioration des mesures à l'aide d'un analyseur ellipsomètre interférentiel. - Il est également possible dans le domaine hertzien d'augmenter considérablement la précision des mesures en améliorant le système analyseur.

En général la lame dont on veut connaître l'indice complexe est horizontale et la source émet une onde polarisée à $45^{\circ}$ du plan vertical (plan d'incidence). Lors de l'incidence principale la vibration réfléchie est elliptique et ses axes sont l'un dans le plan d'incidence (vertical) l'autre perpendiculaire à ce plan. Les méthodes de mesures utilisées consistent soit à chercher le minimum de l'énergie réfléchie polarisée perpendiculairement au plan d'incidence [1], soit à déterminer l'ellipticité minimum en utilisant un ellipsomètre [5] (parfois appelé réflecto-polarimètre). Cet ellipsomètre est composé d'un duplexeur de polarisation dont les directions des « bras », $O Y$ et $O Z$, sont fixées suivant les axes $o y, o z$ (Fig. 2) que la vibration elliptique

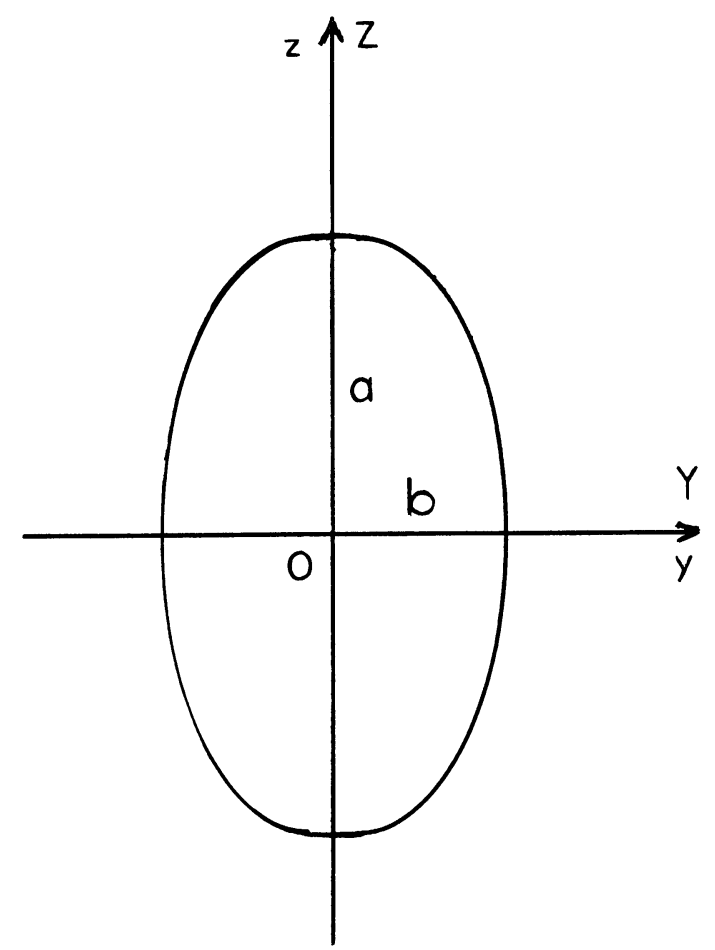

FIG. 2. - $O Y$ et $O Z$ sont les directions des bras du dúplexeur, $o y$ et $o z$ les directions des axes de l'ellipse réfléchie. $O Z$ et $o z$ sont dans le plan d'incidence vertical; oy, $O Y$ suivant une horizontale.

réfléchie doit avoir au moment de l'incidence principale. On recueille donc dans ces conditions à la sortie du duplexeur des amplitudes proportionnelles l'une à $a$, l'autre à $b$ (demi-axes de l'ellipse). On fait alors interférer ces deux composantes après en avoir déphasé une de $\pi / 2$; à l'aide d'un atténuateur calibré on recherche le minimum nul dans le mélangeur. Ces méthodes qui conviennent pour les corps dont l'absorption est importante $[1,5,6,7]$ présentent quelques difficultés pour les corps transparents et peu absorbants qui conduisent à mesurer des ellipticités faibles $(\operatorname{tg} \beta=b / a$ voisine de 0 ) et par suite des atténuations importantes. Il est en effet difficile de réaliser des atténuateurs variables dont le déphasage reste constant, surtout dans le domaine millimétrique.

Mais il existe une autre difficulté plus grave qui n'apparaît pas immédiatement et qui est due au fait que les duplexeurs ne sont pas parfaits et ne séparent 
pas totalement les deux directions orthogonales. En effet les «Turnstiles » qui donnent toute satisfaction par suite du découplage important dans la bande centimétrique $[8,9,10,11]$ sont mécaniquement très difficiles à réaliser au-dessus de $35 \mathrm{GHz}$. Les duplexeurs Fin-Line utilisés en millimétrique ne séparent pas d'une façon suffisante les deux directions orthogonales et présentent de plus des résonances dimensionnelles fonctions de la puissance [12]. J. Mendonça [13] a montré que pour les vibrations ayant une très faible ellipticité, et les vibrations rectilignes, un duplexeur Fin-Line dont les directions des bras coïncident avec les axes de l'ellipse introduisait une erreur sur la mesure des ellipticités ou des angles, qui était de l'ordre de 1,80. Une méthode de compensation a été proposée qui permettrait de réduire cette valeur au demi-degré [14] mais elle est en partie illusoire car si elle respecte la relation énergétique elle ne respecte pas les phases.

La méthode proposée est une transposition au domaine millimétrique de celle, basée sur des « Turnstiles ", déjà utilisée dans le domaine centimétrique [9]. Elle consiste à utiliser le duplexeur en position fixe et à amener le grand axe de la vibration elliptique que l'on veut analyser suivant l'une des bissectrices des bras.

Supposons le dioptre réfléchissant horizontal, le plan d'incidence vertical et la vibration incidente polarisée à $45^{\circ} \mathrm{du}$ plan d'incidence. La vibration elliptique réfléchie dans le cas général est quelconque et son grand axe fait un angle $(\pi / 4-\theta)$ avec le plan d'incidence vertical (Fig. 3). Au moment de l'incidence principale le grand axe de l'ellipse est alors dans le plan d'incidence suivant $o z$. Si le duplexeur est monté de telle sorte que ses bras soient constamment à $45^{\circ}$ du plan d'incidence la vibration elliptique réfléchie ne sera placée suivant la bissectrice qu'au moment de l'incidence principale. Nous allons montrer que, dans ces conditions, on peut déterminer l'incidence principale avec précision dans le cas d'un duplexeur parfait ; mais aussi que dans le cas d'un duplexeur réel, cela permet de réduire considérablement l'erreur due au fait que la séparation des composantes suivant les deux directions orthogonales n'est pas totale.

On remarquera que la recherche du minimum nul dans le cas où les bras du duplexeur sont bissectrices des axes de l'ellipse se fait à l'aide d'un déphaseur ; or l'expérience montre qu'il est plus facile de réaliser un déphaseur dont l'atténuation soit sensiblement constante qu'un atténuateur à déphasage constant, ceci concoure également à augmenter la précision.

Lorsqu'une vibration elliptique dont les amplitudes complexes sont représentées dans le système d'axes $o y_{1}$, $o z_{1}$ (Fig. 3) par la matrice

$$
\left[\begin{array}{c}
a \\
-j \varepsilon b
\end{array}\right]
$$

( $\varepsilon= \pm 1$ suivant que l'ellipse est gauche ou droite) arrive sur un duplexeur de polarisation parfait dont les bras sont $O Z$ et $O Y$; la direction $o y_{1}$ faisant un angle $\theta$

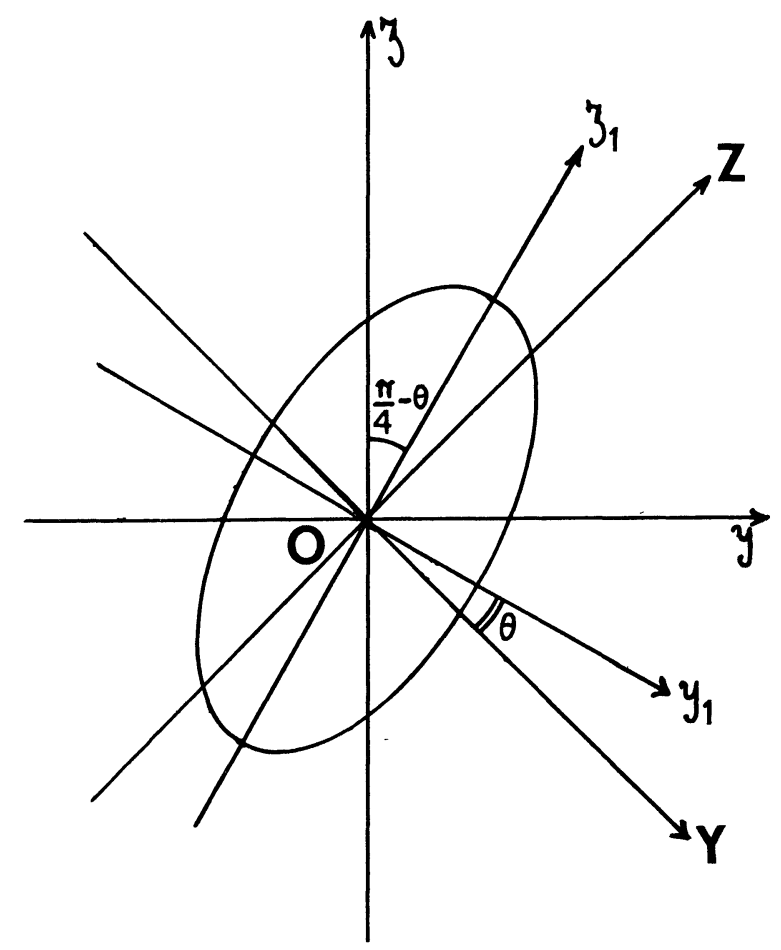

FIG. 3. - $-o y_{1}$ et $o z_{1}$ les axes de la vibration elliptique dans le cas général (angle d'incidence quelconque). $O Y$ et $O Z$ direction des bras du duplexeur à $\pi / 4$ de $o y, o z$, axes de l'ellipse au moment de l'incidence principale. (oz dans le plan vertical d'incidence.)

avec $O Y$, on obtient [9] deux vibrations dont les amplitudes complexes sont proportionnelles à :

$$
\left\{\begin{array}{l}
E_{1}=(a \sin \theta-j \varepsilon b \cos \theta) \\
E_{2}=(a \cos \theta+j \varepsilon b \sin \theta)
\end{array}\right.
$$

qui se transforment classiquement [9] en :

$$
E_{1}=B \mathrm{e}^{-j \varepsilon \psi}
$$

et

$$
E_{2}=A \mathrm{e}^{j \varepsilon \varphi} .
$$

Si on fait interférer ces ondes dans un mélangeur [9] elles prennent l'une par rapport à l'autre un déphasage $\eta$ que l'on pourra modifier à l'aide d'un déphaseur. Nous avons donc :

$$
\begin{aligned}
E & =B \mathrm{e}^{-j \varepsilon \psi}+A \mathrm{e}^{j \varepsilon \varphi} \mathrm{e}^{j \eta} \\
I=E E^{*} & =A^{2}+B^{2}+2 A B \cos [\varepsilon(\psi+\varphi)+\eta]
\end{aligned}
$$

si $A \neq 0$ on peut écrire :

$$
I=A^{2}\left[\frac{B^{2}}{A^{2}}+2 \cos [\varepsilon(\varphi+\psi)+\eta] \frac{B}{A}+1\right] .
$$

Cherchons à quelles conditions $I=0$

$$
\delta^{\prime}=\cos ^{2}[\varepsilon(\psi+\varphi)+\eta]-1=-\sin ^{2}[\varepsilon(\psi+\varphi)+\eta]
$$

donc $\delta^{\prime} \leqslant 0$. Il faut pour obtenir une solution réelle que :

$$
\begin{aligned}
\delta^{\prime}=0 & \Leftrightarrow \sin [\varepsilon(\psi+\varphi)+\eta]=0 \\
& \Leftrightarrow \varepsilon(\psi+\varphi)+\eta=k \pi
\end{aligned}
$$


donc :

$$
\eta=k \pi-\varepsilon(\psi+\varphi)
$$

On a une solution

$$
\frac{B}{A}=-\cos [\varepsilon(\psi+\varphi)+\eta]= \pm 1
$$

Comme on a $\operatorname{tg} \beta=b / a$ avec $0 \leqslant \beta \leqslant \pi / 4$ et $a$ et $b \geqslant 0$ il s'ensuit $A$ et $B \geqslant 0$ donc $A=B$ ce qui conduit à :

$$
\begin{gathered}
a^{2} \cos ^{2} \theta+b^{2} \sin ^{2} \theta=a^{2} \sin ^{2} \theta+b^{2} \cos ^{2} \theta \\
\left(a^{2}-b^{2}\right)\left(\cos ^{2} \theta-\sin ^{2} \theta\right)=0 .
\end{gathered}
$$

Deux cas sont possibles :

1) $a=b$

2) $\cos 2 \theta=0$ soit

$$
\theta=\frac{\pi}{4}+k \frac{\pi}{2} .
$$

Si $a=b$ la vibration est circulaire, sinon elle est elliptique et ses axes sont bissectrices des bras du duplexeur lorsque $I$ est nul. La mesure de l'ellipticité et son signe se déterminent à l'aide du déphasage $\eta$ [9].
Cette ellipticité est liée par des relations classiques [1] à la $\operatorname{tg} \delta$.

Il est donc aisé avec un duplexeur parfait dont les bras sont fixés à $45^{\circ}$ du plan vertical d'incidence de déterminer l'angle d'incidence pour lequel la vibration est réfléchie sous incidence brewstérienne ou principale (les composantes de l'ellipse dans le cas envisagé sont l'une dans le plan vertical l'autre suivant une horizontale) il suffit pour cela de rechercher le minimum nul en faisant varier l'angle d'incidence et le déphasage $\eta$.

Dans le cas où le duplexeur n'est pas parfait et ne sépare pas totalement les deux composantes orthogonales une fraction $k$ des ondes se mélange et un déphasage $\gamma$, que nous supposons identiques pour les deux, s'introduit. Les amplitudes complexes des ondes que l'on retrouve dans chacun des bras du duplexeur sont proportionnelles aux valeurs :

$$
\begin{aligned}
& E_{1}^{\prime}=B \mathrm{e}^{-j \varepsilon \psi}+k A \mathrm{e}^{j \varepsilon \varphi} \mathrm{e}^{j \gamma} \\
& E_{2}^{\prime}=A \mathrm{e}^{j \varepsilon \varphi}+k B \mathrm{e}^{-j \varepsilon \psi} \mathrm{e}^{j \gamma} .
\end{aligned}
$$

Ces ondes interfèrent dans un mélangeur, elles prennent un déphasage $\eta^{\prime}$ qui pourra être modifié comme précédemment

$$
\begin{gathered}
E^{\prime}=\left[A\left(\mathrm{e}^{j \eta^{\prime}}+k \mathrm{e}^{j \gamma}\right) \mathrm{e}^{j \varepsilon \varphi}+B\left(1+k \mathrm{e}^{j\left(\gamma+\eta^{\prime}\right)}\right) \mathrm{e}^{-j \varepsilon \psi}\right] \\
I^{\prime}=A^{2}\left[1+2 k \cos \left(\eta^{\prime}-\gamma\right)+k^{2}\right]+B^{2}\left[1+2 k \cos \left(\eta^{\prime}+\gamma\right)+k^{2}\right]+ \\
+2 A B\left[\cos \left[\eta^{\prime}+\varepsilon(\varphi+\psi)\right]+2 k \cos \gamma \cdot \cos \varepsilon(\varphi+\psi)+k^{2} \cos \left(\eta^{\prime}-\varepsilon(\varphi+\psi)\right)\right] .
\end{gathered}
$$

En remplaçant $A, B, \psi, \varphi$ par leurs valeurs on obtient

d'où

$$
2 A B=\sqrt{\left(a^{2}-b^{2}\right)^{2} \sin ^{2} 2 \theta+4 a^{2} b^{2}}
$$

$$
\begin{aligned}
I^{\prime}=\left\{a^{2}+b^{2}+\sqrt{\left(a^{2}-b^{2}\right)^{2} \sin ^{2} 2 \theta+}\right. & 4 a^{2} b^{2} \\
& +2 k\left\{\left(\eta^{\prime}-\varepsilon(\varphi+\psi)\right]\right\} k^{2}+ \\
& \left.+\sqrt{\left(a^{2}-b^{2}\right)^{2} \sin ^{2} 2 \theta+4 a^{2} b^{2}} \cdot \cos \gamma \cdot \cos \varepsilon(\varphi+\psi)\right\} \\
& +a^{2}+b^{2}+\sqrt{\left(a^{2}-b^{2}\right)^{2} \sin ^{2} 2 \theta+4 a^{2} b^{2}} \cdot \cos \left(\eta^{\prime}+\varepsilon(\varphi+\psi)\right) .
\end{aligned}
$$

On a les inégalités suivantes :

et

$$
\begin{gathered}
\sqrt{\left(a^{2}-b^{2}\right)^{2} \sin ^{2} 2 \theta+4 a^{2} b^{2}} \leqslant \sqrt{\left(a^{2}-b^{2}\right)+4 a^{2} b^{2}}=a^{2}+b^{2} \\
-\left(a^{2}+b^{2}\right) \leqslant \sqrt{\left(a^{2}-b^{2}\right)^{2} \sin ^{2} 2 \theta+4 a^{2} b^{2}} \cdot \cos \left(\eta^{\prime}+\varepsilon(\varphi+\psi)\right) \leqslant a^{2}+b^{2}
\end{gathered}
$$

$$
-\left(a^{2}+b^{2}\right) \leqslant \sqrt{\left(a^{2}-b^{2}\right)^{2} \sin ^{2} 2 \theta+4 a^{2} b^{2}} \cdot \cos \left(\eta^{\prime}-\varepsilon(\varphi+\psi)\right) \leqslant a^{2}+b^{2}
$$

ce qui conduit à :

et

$$
a^{2}+b^{2}+\sqrt{\left(a^{2}-b^{2}\right)^{2} \sin ^{2} 2 \theta+4 a^{2} b^{2}} \cdot \cos \left(\eta^{\prime}-\varepsilon(\varphi+\psi)\right) \geqslant 0
$$

$$
a^{2}+b^{2}+\sqrt{\left(a^{2}-b^{2}\right)^{2} \sin ^{2} 2 \theta+4 a^{2} b^{2}} \cdot \cos \left(\eta^{\prime}+\varepsilon(\varphi+\psi)\right) \geqslant 0 .
$$

D'autre part, on remarque que :

1) Si $\theta= \pm \pi / 4$ et si $\cos \left(\eta^{\prime}-\varepsilon(\varphi+\psi)\right)=-1$ le terme en $k^{2}$ et le terme en $k$ s'annulent.

2) Si $\theta= \pm \pi / 4$ et si $\cos \left(\eta^{\prime}+\varepsilon(\varphi+\psi)\right)=-1$ le terme indépendant de $k$ et le terme en $k$ s'annulent.
Compte tenu des inégalités et des remarques précédentes la somme des termes 1 et 3 est toujours positive puisqu'ils ne s'annulent pas en même temps. La solution $I^{\prime}=0$ ne peut s'obtenir que lorsque le second terme est négatif.

La remarque $n^{0} 2$ est identique à celle trouvée dans le 
cas d'un duplexeur parfait. Donc au terme du second ordre près on obtiendra le minimum nul pour les mêmes conditions qu'avec un duplexeur parfait.

Dans le cas de l'exemple considéré : duplexeur FinLine ayant une directivité de $30 \mathrm{~dB}$ il est aisé de chiffrer l'erreur pour diverses valeurs de l'ellipticité.

Il est remarquable toutefois que dans le cas d'une vibration rectiligne (cas d'un diélectrique non absorbant) il n'y a aucune erreur due au manque de directivité du duplexeur considéré.

En effet si $b=0$ ceci entraîne :

$$
\begin{aligned}
& \sin \varphi=0 \Rightarrow \varphi=k_{1} \pi \\
& \sin \psi=0 \Rightarrow \psi=k_{2} \pi
\end{aligned}
$$

donc

$$
\varepsilon(\psi+\varphi)=k \pi .
$$

Deux cas sont à envisager

$$
\sin 2 \theta=+1
$$

on obtient alors $\theta=\pi / 4+k \pi / 2$; et

$$
\begin{aligned}
\frac{I^{\prime}}{a^{2}}=k^{2}[1+\cos ( & \left.\left.\eta^{\prime}-k \pi\right)\right]+ \\
& +2 k\left[\cos \eta^{\prime} \cos \gamma+\cos k \pi \cos \gamma\right] \\
& +1+\cos \left(\eta^{\prime}+k \pi\right) .
\end{aligned}
$$

La valeur de $I^{\prime}$ sera nulle pour

$$
\begin{aligned}
& \cos \left(\eta^{\prime}-k \pi\right)=-1 \\
& \cos \eta^{\prime}=-\cos k \pi \\
& \cos \left(\eta^{\prime}+k \pi\right)=-1 .
\end{aligned}
$$

Deux cas sont à nouveau à envisager :

1) $k$ pair qui conduit à $\cos \eta^{\prime}=-1$ donc $\eta^{\prime}=\pi$.
2) $k$ impair qui donne $\cos \eta^{\prime}=+1$ donc $\eta^{\prime}=0$. Le cas où $\sin 2 \theta=-1$ conduit à des résultats symétriques.

Donc dans le cas du pointé d'une vibration rectiligne aucune erreur systématique ne s'introduit par le fait que le duplexeur n'est pas parfait avec la méthode proposée (la vibration est bissectrice des bras). Par contre si la vibration est suivant l'un des bras (méthode classique) en plus de l'erreur de mesure due à l'atténuateur il y a pour un découplage de $30 \mathrm{~dB}$ entre les bras une erreur qui est dans le meilleur des cas de $\frac{1}{2}$ degré [13, 14]. Ceci est important, la précision, dans le cas du montage proposé, est seulement limitée par les appareils de détection, etc...

Il convient également de remarquer que dans le cas d'un duplexeur Fin-Line qui présente des résonances dimensionnelles variables avec la puissance [12] le montage proposé qui envoie la même énergie dans chacun des bras est préférable.

Il est à souhaiter que l'utilisation de ce montage se généralise chaque fois qu'il y a lieu de pointer l'azimut d'une vibration elliptique, d'autant qu'il permet, de plus, la mesure de l'ellipticité avec son signe.

On pourrait montrer que l'analyseur-ellipsomètre interférentiel précédent permet aussi la mesure de la dépolarisation des ondes [15].

Ainsi donc les mesures de permittivité complexe sous incidence brewstérienne ou principale peuvent être convenablement améliorées, d'une part, en évitant les réflexions multiples et, d'autre part, en utilisant un analyseur de vibration constitué d'un duplexeur placé à $45^{\circ}$ des axes de la vibration elliptique obtenue au moment de l'incidence principale. La mesure de l'ellipticité à l'aide de $\eta$ permettrait de déterminer de manière classique la valeur de la tangente $\delta$.

\section{Bibliographie}

[1] Charru, A. et Bretenoux, A., C. R. Hebd. Séan. Acad. Sci. 263 (1966) Série B, 45.

[2] Born, M. et Wolf, E., Principles of Optics (Pergamon Press, N. Y.) 1959.

[3] SARdos, R., Electronica y Fisica Aplicada 16 (1973) 156.

[4] Sardos, R., Brevet ANVAR no 7319248.

[5] Charru, A., Bretenoux, A., Sarremejean, A. et Urbain, G., Revue Phys. Appl. 4 (1969) 37.

[6] Ohebsian, D., Thèse de $3^{\mathrm{e}}$ cycle, Bordeaux 1971.

[7] Hua-Ngoc-Trung, Thèse de $3^{\mathrm{e}}$ cycle, Bordeaux 1973.
[8] Soutif, J., Thèse Grenoble 1957.

[9] SARdos, R., Thèse Bordeaux 1965.

[10] BISSEY, J. C., Thèse de 3e cycle, Bordeaux 1969.

[11] HAYE, K., Phys. Rev. (à paraître).

[12] Heald, M. A. et Wharton, C. B., Plasma Diagnostics With Microwaves (John Wiley and Sons inc. N. Y.) 1965.

[13] Mendonca, J. T., Thèse de $3^{\mathrm{e}}$ cycle, Paris 1973.

[14] Vernon, R. J. et Durschner, T. A., I. E. E. E. Trans. Microwave Theory Tech. 19 (1971) 287.

[15] Sardos, R., J. Appl. Phys. (à paraître). 